\title{
Multi-resolution Joint LBP Histograms for Biomedical Image Retrieval
}

\author{
K. Prasanthi Jasmine \\ Department of Electronics and Communication \\ Engineering \\ Andhra University, Visakhapatnam \\ Andhra Pradesh, India
}

\author{
P. Rajesh Kumar \\ Department of Electronics and Communication \\ Engineering \\ Andhra University, Visakhapatnam \\ Andhra Pradesh, India
}

\begin{abstract}
In this paper, a new image indexing and retrieval algorithm using multi-resolution local binary patterns (LBP) with joint histogram is proposed. The existing LBP extracts the relationship between the center pixel and its surrounding neighbors in an image. The proposed method encodes the joint histogram between the multiresolution LBPs which are calculated using Gaussian filter bank with different standard deviations. The retrieval results of the proposed method have been tested on OASIS magnetic resonance imaging (MRI) database. The results after being investigated shows a significant improvement in terms of precision as compared to LBP and other LBP like features.
\end{abstract}

\section{Keywords}

Local Binary Patterns (LBP); Texture; Pattern Recognition; Feature Extraction; Biomedical Image Retrieval.

\section{INTRODUCTION}

With the growth in medical technology and advancement of the living world, there has been an expansion of biomedical images in hospitals and medical institutions in order to meet ones' medical requirement. This huge data is in different format such as computer tomography (CT), magnetic resonance images (MRI), ultrasound (US), X-ray etc. Handling of this data by human annotation is a cumbersome task thereby, arousing a dire need for some familiar search technique i. e. content based image retrieval (CBIR). It is very difficult to identify the exact disease location in the patient reports (images) for new physicians as compared with more experienced physicians. This problem can solve using CBIR system by giving that patient report as a query and the doctor will retrieve related patient reports which are previously collected and stored with description about disease in the database. With the help of old related reports, the doctors can identify the exact disease in the present patient report. The previously available CBIR systems for medical image retrieval are available in [1-4].

The feature extraction forms a prominent stair in CBIR and its effectiveness relies typically on the method of features extraction from raw images. Comprehensive and extensive literature survey on CBIR is presented in [5-10].

Texture is used to specify the roughness or coarseness of object surface and described as a pattern with some kind of regularity. Many researchers have put forward various algorithms for texture analysis, such as the gray co-occurrence matrixes [10], Markov random field (MRF) model [11], simultaneous auto-regressive
(SAR)model [12], Wold decomposition model [13], Gabor filtering $[14,15]$ and wavelet decomposition $[16,17]$ and so on. Subrahmanyam et al. [18] combined the color (color histogram) and texture (wavelet transform) features for CBIR. Subrahmanyam et al. proposed correlogram algorithm for image retrieval using wavelets and rotated wavelets (WC+RWC) [19].

Ojala et al. proposed the local binary patterns (LBP) for texture description [20] and these LBPs are converted to rotational invariant for texture classification [21]. pietikainen et al. proposed the rotational invariant texture classification using feature distributions [22]. Ahonen et al. [23] and Zhao et al [24] used the LBP operator facial expression analysis and recognition. Heikkila et al. proposed the background modeling and detection by using LBP [25]. Huang et al. proposed the extended LBP for shape localization [26]. Heikkila et al. used the LBP for interest region description [27]. Li et al. used the combination of Gabor filter and LBP for texture segmentation [28]. Zhang et al. proposed the local derivative pattern for face recognition [29]. They have considered LBP as a nondirectional first order local pattern, which are the binary results of the first-order derivative in images. B. Zhang et al. [30] have proposed the directional binary code (DBC) for face recognition. The DBC is encodes the directional edge information in a neighborhood.

Subrahmanyam et al. have proposed the directional binary wavelet patterns (DBWP) [31] for biomedical image retrieval. The 8-bit grayscale image is divided into eight binary bit-planes, and then binary wavelet transform (BWT) which is similar to the lifting scheme in real wavelet transform (RWT) is performed on each bitplane to extract the multi-resolution binary images. The LBP features are extracted from the resultant BWT sub-bands.

To improve the retrieval performance in terms of retrieval accuracy, in this paper, we calculated the joint histogram between the multiresolution LBPs using Gaussian filter banks. The performance of the proposed method has been tested on Open Access Series of Imaging Studies (OASIS) MRI database for proving the worth of our algorithm. The results after investigation show a significant improvement in terms of their precision as compared to LBP, LBP with Gabor transform and DBWP.

The organization of the paper as follows: In section 1, a brief review of image retrieval and related work is given. Section 2, 3 and 4 presents a concise review of local binary patterns, multi-resolution LBPs and the proposed system framework respectively. Experimental results and discussions are given in section 5. Based on above work conclusions are derived in section 6 . 


\section{LOCAL BinARY PATTERNS}

The LBP operator was introduced by Ojala et al. [20] for texture classification. Success in terms of speed (no need to tune any parameters) and performance is reported in many research areas such as texture classification, face recognition, object tracking, bio-medical image retrieval and finger print recognition.

Given a center pixel in the $3 \times 3$ pattern, LBP value is computed by comparing its gray scale value with its neighborhoods based on Eq. (1) and Eq. (2):

$$
\begin{aligned}
& L B P_{P, R}=\sum_{i=1}^{P} 2^{(i-1)} \times f\left(I\left(g_{i}\right)-I\left(g_{c}\right)\right) \\
& f(x)= \begin{cases}1 & x \geq 0 \\
0 & \text { else }\end{cases}
\end{aligned}
$$

where $I\left(g_{c}\right)$ denotes the gray value of the center pixel, $I\left(g_{i}\right)$ is the gray value of its neighbors, $P$ stands for the number of neighbors and $R$, the radius of the neighborhood.

Fig. 1 shows an example of obtaining an LBP from a given $3 \times 3$ pattern. The histograms of these patterns extract the distribution of edges in an image [20].
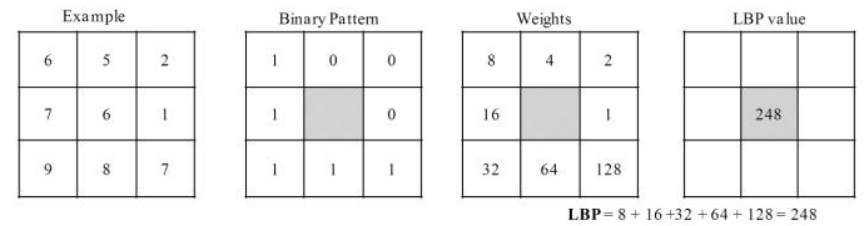

Fig. 1: LBP calculation for $3 \times 3$ pattern

\section{MULTI-RESOLUTION LBPS}

\subsection{Gaussian Filter Bank}

The 1-D Gaussian function is defined by

$$
g(t, \sigma)=\frac{1}{\sqrt{2 \pi \sigma}} e^{-t^{2} / 2 \sigma^{2}}
$$

where the parameter $\sigma$ denotes the Gaussian half-width. Fig. 2 displays this Gaussian function and its $1^{\text {st }}$ and $2^{\text {nd }}$ derivatives for $\sigma$ $=1$.

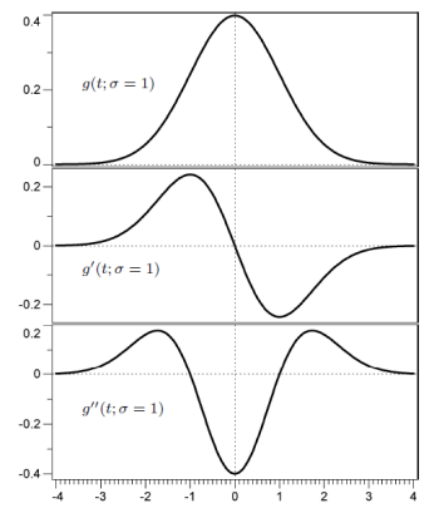

Fig. 2: The Gaussian function $g(t, \sigma=1)$ and its derivatives.

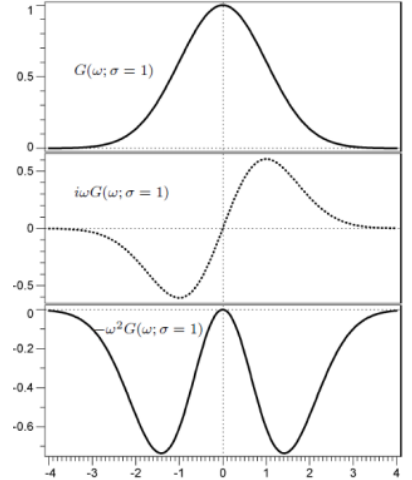

Fig. 3: The Fourier transforms of the Gaussian function $g(t, \sigma=1)$ and its $\mathbf{1}^{\text {st }}$ and $2^{\text {nd }}$ derivatives. The Fourier transform of the Gaussian $1^{\text {st }}$ derivative $g^{\prime}(t, \sigma)$ is purely imaginary, as indicated here by the dashed curve.

The Fourier transform of the Gaussian function is also a Gaussian:

$$
G(\omega, \sigma)=\int_{-\infty}^{\infty} d t e^{-i \omega t} g(t, \sigma)=e^{-\omega^{2} \sigma^{2} / 2}
$$

Fig. 3 displays this Fourier transform and those of the $1^{\text {st }}$ and $2^{\text {nd }}$ Gaussian derivatives for $\sigma=1$. Eq. (4) imply that convolution of a signal with a Gaussian is equivalent to low-pass filtering of that signal.

The 2-D circular Gaussian function is defined as:

$$
g(x, y, \sigma)=\frac{1}{2 \pi \sigma^{2}} e^{-\left(x^{2}+y^{2}\right) / 2 \sigma^{2}}
$$

The Gaussian images of a given image are calculated as follows:

$$
L(x, y, \sigma)=g(x, y, \sigma) * I(x, y)
$$

where $\sigma$ is different scales and (*) indicates the convolution operator.

After calculating the three multi-resolution images for a given image we perform the LBP operation on each image. To reduce the feature dimension we selected the rotational invariant uniform two patterns for LBP calculation.

The uniform pattern refers to the uniform appearance pattern which has limited discontinuities in the circular binary presentation. In this paper, the pattern which has less than or equal to two discontinuities in the circular binary presentation is considered as the uniform pattern and remaining patterns considered as non-uniform patterns.

Fig. 4 shows all uniform patters for $P=8$. The distinct values for given query image is $P(P-1)+3$ by using uniform patterns. But these features are not rotational invariant.

The rotational invariant patterns $\left(L B P_{P, R}^{\text {riu2 }}\right)$ can be constructed by adding all eight patterns in the each row of Fig. 4 as shown in Fig. 5 . The distinct values for a given query image is $P+2$ by using rotational invariant patterns $\left(L B P_{P, R}^{\text {riu2 }}\right)$.

After collecting the multi-resolution LBPs we calculated the joint histogram between them for feature extraction. Hence, the feature vector length of the proposed method at $\mathrm{P}=8$ is $10 \times 10 \times 10(=1000)$. 


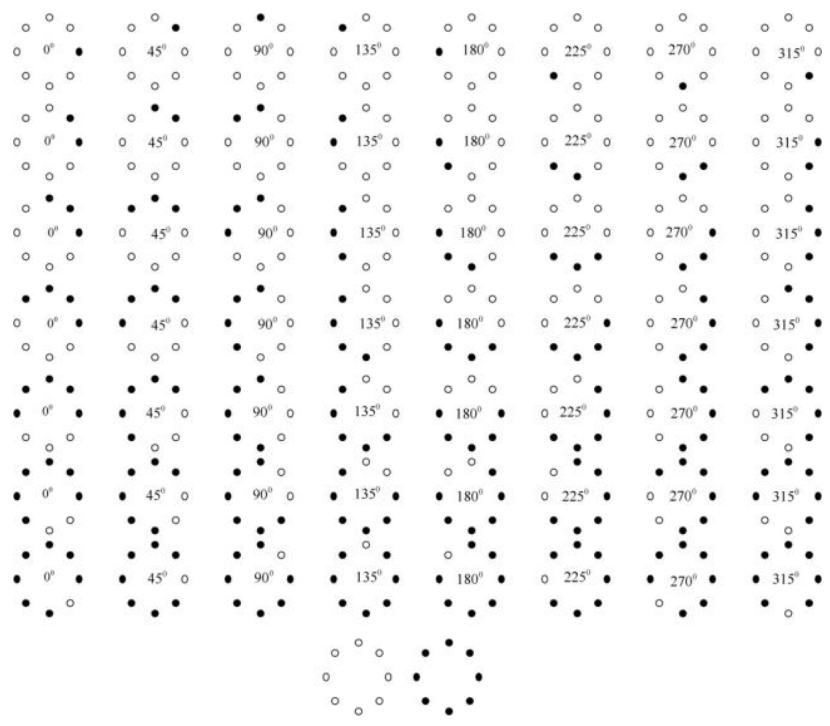

Fig. 4: Uniform patters when $P=8$. The black and white dots represent the bit values of 1 and 0 in the LMEBP operator

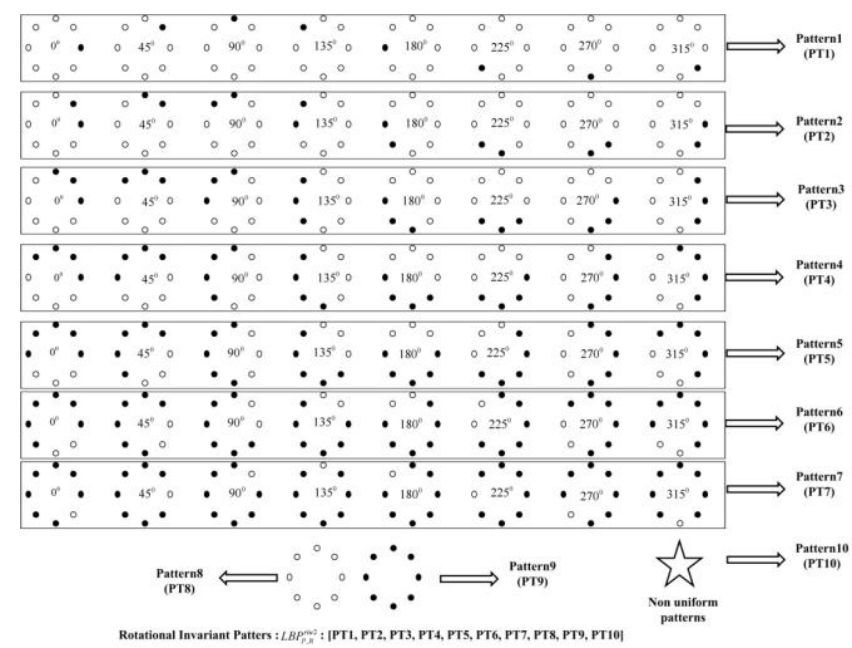

Fig. 5: Rotational variant LMEBP patterns are converted into rotational invariant LMEBP patterns

\section{PROPOSED SYSTEM FRAMEWORK}

In this paper, we proposed the new technique by calculating the joint histogram between the multi-resolution rotational invariant LBP subimages. Finally, feature vector is constructed by concatenating the features collected using joint histograms.

Algorithm:

Input: Image; Output: Retrieval results.

1. Load the input image.

2. Calculate the three multi-resolution images using Gaussian filter bank.

3. Calculate the LBP on each multi-resolution image and make them into rotational invariant.

4. Calculate the joint histogram between them.

5. Form the feature vector by concatenating histograms.

6. Calculate the best matches using Eq. (7).
7. Retrieve the number of top matches.

\subsection{Similarity Measurement}

In the presented work $d_{1}$ type of similarity distance metric is used as shown below:

$D_{1}$ Distance

$$
D(Q, T)=\sum_{i=1}^{L g}\left|\frac{f_{T, i}-f_{Q, i}}{1+f_{T, i}+f_{Q, i}}\right|
$$

where $Q$ is query image, $L g$ is feature vector length, $T$ is image in database; $f_{I, i}$ is $i^{\text {th }}$ feature of image $I$ in the database, $f_{Q, i}$ is $i^{\text {th }}$ feature of query image $Q$.

\section{EXPERIMENTAL RESULTS AND DISCUSSION}

In order to analyze the performance of our proposed method for image retrieval the experiment is conducted on MRI medical image database. Results obtained are discussed in the following subsections.

The abbreviations for extracted features are given below:

LBP: well-known LBP features

GLBP: LBP with Gabor transform

DBWP: Directional Binary Wavelet Patterns

Joint_LBP_Mu: Joint multi-resolution LBP histograms.

In this experiment, each image in the database is used as the query image. For each query, the system collects the $n$ database images $X=\left(x_{1}, x_{2}, \ldots \ldots \ldots, x_{n}\right)$ with the shortest image matching distance is given by Eq. (7). If $x_{i} ; i=1,2, \ldots . n$ belong to the same category of the query image, we say the system has correctly match the desired, otherwise the system has failed.

The average precision judges the performance of the proposed method which is shown below:

For the query image $I_{q}$, the precision is defined as follows:

$$
P\left(I_{q}, n\right)=\frac{1}{n} \sum_{i=1}^{|D B|}\left|\delta\left(\Phi\left(I_{i}\right), \Phi\left(I_{q}\right)\right)\right| \operatorname{Rank}\left(I_{i}, I_{q}\right) \leq n \mid
$$

where ' $n$ ' indicates the number of retrieved images, $|D B|$ is size of image database. $\Phi(x)$ is the category $(G)$ of ' $x$ ', $\operatorname{Rank}\left(I_{i}, I_{q}\right)$ returns the rank of image $I_{i}$ (for the query image $I_{q}$ ) among all images of $|D B|$ and $\delta\left(\Phi\left(I_{i}\right), \Phi\left(I_{q}\right)\right)=\left\{\begin{array}{ll}1 & \Phi\left(I_{i}\right)=\Phi\left(I_{q}\right) \\ 0 & \text { Otherwise }\end{array}\right.$.

The Open Access Series of Imaging Studies (OASIS) [32] is a series of magnetic resonance imaging (MRI) dataset that is publicly available for study and analysis. This dataset consists of a cross-sectional collection of 421 subjects aged 18 to 96 years. The MRI acquisition details are given in Table 1 .

For image retrieval purpose we grouped these 421 images into four classes based on the shape of ventriculars in the images. Fig. 6 depicts the sample images of OASIS database (one image from each category). 
Table 1: MRI data acquisition details [32]

\begin{tabular}{|c|c|}
\hline Sequence & $\begin{array}{c}\text { MP- } \\
\text { RAGE }\end{array}$ \\
\hline TR (msec) & 9.7 \\
\hline TE (msec) & 4.0 \\
\hline Flip angle (o) & 10 \\
\hline TI (msec) & 20 \\
\hline TD (msec) & 200 \\
\hline Orientation & Sagittal \\
\hline $\begin{array}{c}\text { Thickness, gap } \\
\text { (mm) }\end{array}$ & $1.25,0$ \\
\hline $\begin{array}{c}\text { Resolution } \\
\text { (pixels) }\end{array}$ & $176 \times 208$ \\
\hline
\end{tabular}
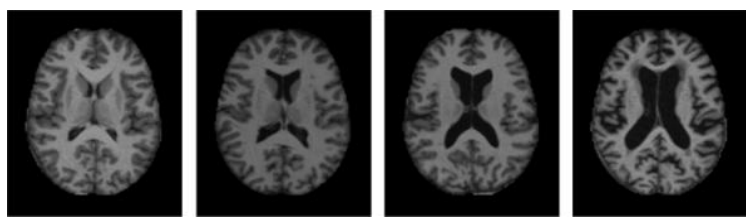

Fig. 6: Sample images from OASIS database (one image per category)

Table 2: Results of all techniques in terms of Precision on OASIS Database

\begin{tabular}{|c|c|c|c|c|c|}
\cline { 2 - 6 } \multicolumn{1}{c|}{ PM: Joint_LBP_Mu } \\
\cline { 2 - 6 } & \multicolumn{5}{c|}{ Precision (\%) $(\boldsymbol{n = 1 0})$} \\
\cline { 2 - 6 } & Group & Group & Group & Group & \\
$\mathbf{1}$ & $\mathbf{2}$ & $\mathbf{3}$ & $\mathbf{4}$ & Total \\
\hline LBP & 51.77 & 32.54 & 33.82 & 49.06 & 42.63 \\
\hline GLBP & 54.43 & 37.94 & 26.51 & 46.03 & 42.42 \\
\hline DBWP & 52.74 & 37.74 & 34.38 & 60.00 & 47.05 \\
\hline PM & $\mathbf{5 9 . 5 9}$ & $\mathbf{4 5 . 4 9}$ & $\mathbf{3 9 . 2 0}$ & $\mathbf{5 0 . 0 9}$ & $\mathbf{4 8 . 0 7}$ \\
\hline
\end{tabular}

$n$-Number of top matches considered

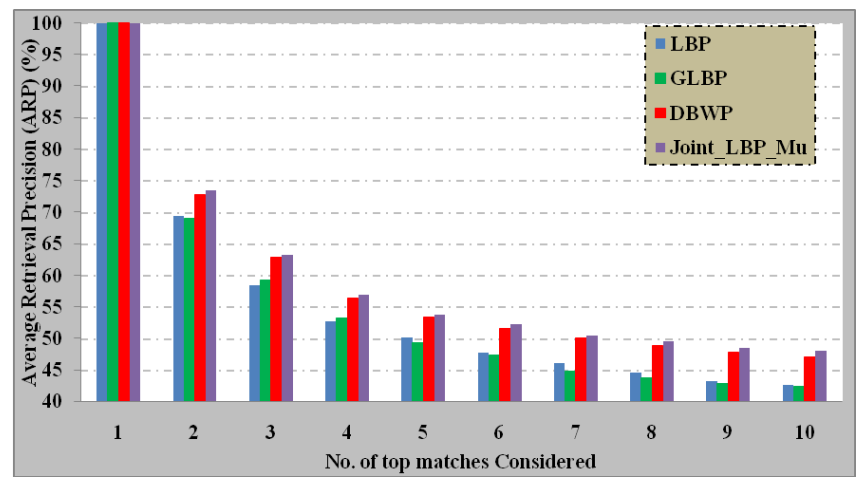

Fig. 7: Comparison of proposed method (Joint_LBP_Mu) with the other existing methods as a function of number of top matches considered on OASIS database

Table 2 shows the performance of various methods in terms of precision on OASIS-MRI database. Fig. 7 illustrates the performance of various methods in terms of average retrieval precision as a funtion of number of top matches considered. From Table 2 and Fig. 7, it clear that the proposed method shows a better performance as compared to other existing methods in terms of precision on OASIS-MRI database. Fig. 8 illustrates the query results of the proposed method on OASIS-MRI database.

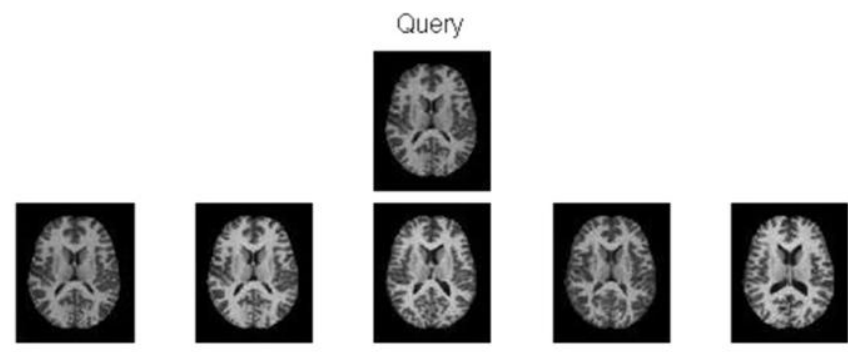

(a)

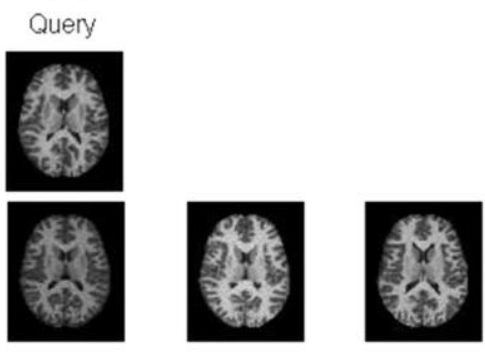

(b)

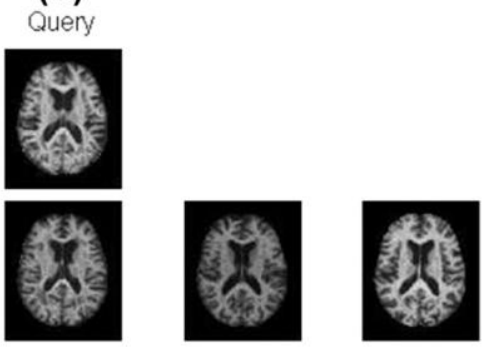

(c)

Fig. 8: Query results of the proposed method on OASIS-MRI database.

\section{CONCLUSIONS}

A new image indexing and retrieval algorithm is proposed in this paper by calculating the joint histogram between the multiresolution subimages. The multi-resolution images are calculated using Gaussian filter bank at different standard deviation. The experimentation has been carried out on OASIS-MRI database for proving the worth of our algorithm. The results after being investigated show a significant improvement in terms of their evaluation measures as compared to LBP, GLBP and DBWP features.

\section{ACKNOWLEDGEMENT}

Our sincere thanks to Dr. Subramanyam Murala, Post-Doc. Fellow, University of Windsor, Windsor, ON, Canada for providing the OASIS-MRI database for evaluation of our method for biomedical image retrieval. Acknowledgement

\section{REFERENCES}

[1] A. Mueen, R. Zainuddin, and M. Sapiyan Baba, MIARS: A Medical Image Retrieval System. J. Med. Syst., 34 859-864, 2010.

[2] Chu, W., Hsu, C., Cardenas, C., and Taira, R., Aknowledgebased image retrieval with spatial and temporal constructs. IEEE Trans. Knowl. Data Eng. 10 (6) 872-888, 1998. 
[3] Shyu, C., Kak, A., Kosaka, A., Aisen, A., and Broderick, L., ASSERT: A physician-in-the-loop content-based inage retrieval system for HRCT image databases. Comput. Vis. Image Underst. 75 111-132, 1998.

[4] Müller, H., Lovis, C., and Geissbuhler, A., Medical Image retrieval-the MedGIFT project. Medical Imaging and telemedicine, 2-7, 2005.

[5] Y. Rui and T. S. Huang, Image retrieval: Current techniques, promising directions and open issues. J. Vis. Commun. Image Represent., 10 39-62, 1999.

[6] A. W.M. Smeulders, M. Worring, S. Santini, A. Gupta, and R. Jain, Content-based image retrieval at the end of the early years. IEEE Trans. Pattern Anal. Mach. Intell., 22 (12) 13491380, 2000.

[7] M. Kokare, B. N. Chatterji, P. K. Biswas, A survey on current content based image retrieval methods. IETE J. Res., 48 (3\&4) 261-271, 2002.

[8] M. S. Lew, N. Sebe, C. Djerba, and R. Jain, Content-based multimedia information retrieval: state of the art and challenges. ACM Trans. Multimedia Comput., Commun., Appl., 2 (1) 1-19, 2006.

[9] Ying Liu, Dengsheng Zhang, Guojun Lu, Wei-Ying Ma, Asurvey of content-based image retrieval with high-level semantics. J. Pattern Recognition, 40 262-282, 2007.

[10] H. M“uller, N. Michoux, D. Bandon, and A. Geisbuhler, A review of content-based image retrieval systems in medical applications-Clinical benefits and future directions. J. Med. Inf., 73 (1) 1-23, 2004.

[11] G. Cross, A. Jain, Markov random field texture models, IEEE Trans. Pattern Anal. Mach. Intell. 5 (1) (1983) 25-39.

[12] J. Mao, A. Jain, Texture classification and segmentation using multi-resolution simultaneous autoregressive models, Pattern Recognition 25 (2) (1992) 173-188.

[13] F. Liu, R. Picard, Periodicity, directionality, and randomness: wold features for image modeling and retrieval, IEEE Trans. Pattern Anal. Mach. Intell. 18 (7) (1996) 722-733.

[14] B.S. Manjunath, W.Y. Ma, Texture features for browsing and retrieval of image data, IEEE Trans. Pattern Anal. Mach. Intell. 18 (8) (1996) 837-842.

[15] J. Han, K.-K. Ma, Rotation-invariant and scale-invariant Gabor features for texture image retrieval, Image Vision Comput. 25 (2007) 1474-1481.

[16] T. Chang, C.C. Jay Kuo, Texture analysis and classification with tree-structured wavelet transform, IEEE Trans. Image Process. 2 (4) (1993) 429-441.

[17] A. Laine, J. Fan, Texture classification by wavelet packet signatures, IEEE Trans. Pattern Anal. Mach. Intell. 11 (15) (1993) 1186-1191.

[18] M. Subrahmanyam, A. B. Gonde and R. P. Maheshwari, Color and Texture Features for Image Indexing and Retrieval, IEEE Int. Advance Computing Conf., Patial, India, (2009) 1411-1416.
[19] Subrahmanyam Murala, R. P. Maheshwari, R. Balasubramanian, A Correlogram Algorithm for Image Indexing and Retrieval Using Wavelet and Rotated Wavelet Filters, Int. J. Signal and Imaging Systems Engineering.

[20] T. Ojala, M. Pietikainen, D. Harwood, A comparative sudy of texture measures with classification based on feature distributions, Elsevier J. Pattern Recognition, 29 (1): 51-59, 1996.

[21] T. Ojala, M. Pietikainen, T. Maenpaa, Multiresolution grayscale and rotation invariant texture classification with local binary patterns, IEEE Trans. Pattern Anal. Mach. Intell., 24 (7): 971-987, 2002.

[22] M. Pietikainen, T. Ojala, T. Scruggs, K. W. Bowyer, C. Jin, K. Hoffman, J. Marques, M. Jacsik, W. Worek, Overview of the face recognition using feature distributions, Elsevier $\mathrm{J}$. Pattern Recognition, 33 (1): 43-52, 2000.

[23] T. Ahonen, A. Hadid, M. Pietikainen, Face description with local binary patterns: Applications to face recognition, IEEE Trans. Pattern Anal. Mach. Intell., 28 (12): 2037-2041, 2006.

[24] G. Zhao, M. Pietikainen, Dynamic texture recognition using local binary patterns with an application to facial expressions, IEEE Trans. Pattern Anal. Mach. Intell., 29 (6): 915-928, 2007.

[25] M. Heikkil;a, M. Pietikainen, A texture based method for modeling the background and detecting moving objects, IEEE Trans. Pattern Anal. Mach. Intell., 28 (4): 657-662, 2006.

[26] X. Huang, S. Z. Li, Y. Wang, Shape localization based on statistical method using extended local binary patterns, Proc. Inter. Conf. Image and Graphics, 184-187, 2004.

[27] M. Heikkila, M. Pietikainen, C. Schmid, Description of interest regions with local binary patterns, Elsevie J. Pattern recognition, 42: 425-436, 2009.

[28] M. Li, R. C. Staunton, Optimum Gabor filter design and local binary patterns for texture segmentation, Elsevie J. Pattern recognition, 29: 664-672, 2008.

[29] B. Zhang, Y. Gao, S. Zhao, J. Liu, Local derivative pattern versus local binary pattern: Face recognition with higherorder local pattern descriptor, IEEE Trans. Image Proc., 19 (2): 533-544, 2010.

[30] B. Zhang, L. Zhang, D. Zhang, L. Shen, Directional binary code with application to PolyU near-infrared face database, Pattern Recognition Letters 31 (2010) 2337-2344.

[31] Subrahmanyam Murala, R. P. Maheshwari, R. Balasubramanian, "Directional Binary Wavelet Patterns for Biomedical Image Indexing and Retrieval," Journal of Medical Systems, 36 (5) 2865-2879

[32] D. S.Marcus, T. H. Wang, J. Parker, J. G. Csernansky, J. C.Morris, and R. L. Buckner, Open access series of imaging studies (OASIS): Crosssectional MRI data in young, middle aged, nondemented, and demented older adults. J. Cogn. Neurosci., 19 (9) 1498-1507, 2007. 